\title{
Ultrastrong and excellent dynamic mechanical properties of carbon nanotube
} composites

Dongmei $\mathrm{Hu}^{1,2}$, Yajuan Xing ${ }^{2,3}$, Minghai $\mathrm{Chen}^{2}$, Bohong $\mathrm{Gu}^{1}$, Baozhong Sun ${ }^{1 *}$, Qingwen $\mathrm{Li}^{2 *}$

1 College of Textiles, Key Laboratory of High Performance Fibers \&Products, Ministry of Education, Donghua University, Shanghai, China

2 Suzhou Institute of Nano-Tech and Nano-Bionics, Key Laboratory of Nanodevices and Applications, Chinese Academy of Sciences, Suzhou 215123, China;

3 Aerospace Research Institute of Materials and Processing Technology,Beijing,

China

*Corresponding author: sunbz@dhu.edu.cn (B. Sun); qwli2007@sinano.ac.cn (Q. Li)

\begin{abstract}
High performance carbon nanotube (CNT)/polyimide (PI) composites with high CNT fraction and high degree of orientation of CNTs were fabricated by infiltration-winding method by coupling PI solution with continuous horizontal aligned few walled CNT film transferred from the vertically CNT array using layer by layer assembly. Different CNT fractions of CNT/PI composites were obtained by tuning the concentration of PI solution. The static and dynamic mechanical properties of CNT/PI composites were investigated. The results show that the interaction between CNTs was greatly improved by the enhancement of contact area of CNTs through PI solution shrink the CNT film, thus the strength and modulus of CNT/PI composites were significantly enhanced up to $3.9 \mathrm{GPa}$ and $182 \mathrm{GPa}$, respectively. The dynamic mechanical analysis (DMA) results showed that the glass transition temperature of PI increased over $130{ }^{\circ} \mathrm{C}$ up to $325{ }^{\circ} \mathrm{C}$ due to the nanometer interface interaction between $\mathrm{CNT}$ and PI resin matrix. The CNT/PI composites also demonstrated excellent structure stability. The excellent mechanical properties make the composites promising for high temperature structural and functional materials for aerospace applications.
\end{abstract}

Keywords: Carbon nanotube; Polyimide; Composites; Dynamic mechanical 
properties

\section{Introduction}

Nanocomposites have become a new growth point of advanced composite technology, is also one of the most active research areas. Extraordinary features of nanocomposite materials make them have broad applications in the aerospace and other fields [1-3]. Since the discovery of carbon nanotube (CNT), it combines with small diameter, large aspect ratio and its special structure produces exceptional properties [4-10], such as excellent electrical, mechanical, thermal, and magnetic properties, high surface areas, and flexible and high chemical stability, and therefore CNT is considered to be the most promising candidates of carbon fiber as next generation of reinforce materials. Therefore, so many advantages make carbon nanotubes become a promising alternative for carbon fiber to meet the challenges of the next generation aerospace technologies. CNTs are usually used as reinforcing fillers embedded in a polymer matrix to confer the CNT's inherent properties of strength, stiffness, thermal and electrical conductivity to the composite. The most common and traditional method of CNT modified composites usually disperse CNTs in the polymer matrix, whose fraction in their composites is usually lower than $10 \mathrm{wt} \%$ and have random distribution with no orientation. Due to the dispersion technology it results in low CNT fraction and poor alignment, so the superior performance of individual CNT tubes is not well represented in the CNT/polymer composites. Various approaches [11-15] were tried to improve the performance of CNT/polymer composites, such as CNT functionalization, the alignment improvement through magnetic field process, improved CNT disperse station through new fabrication such as the three roll mill process, increase CNT loading fraction [16,17]. The floating catalysts was a better methods to prepared continuous CNT fiber, film and composites. [18-20], but network architectures of carbon nanotubes decrease orientation degree causing the limited improve for carbon nanotube composites mechanical properties. However, a variety of methods were applied to improve the properties of composites, and indeed improved, but it was still far below that of the individual CNTs. 
Recently, inspiring from the CNT array based spinning method of CNT fiber fabrication, a novel approach had been developed for producing high performance CNT composites. A continuous CNT film was drawn from the vertically aligned CNT array and wound onto the rotating mandrel. During the drawing process, a polymer solution was sprayed onto the single layer film after winding and then stacked layer by layer. Due to the capillary force of solution CNT film shrunk and CNT were closely packed in the thickness direction. The high packing density can achieve a large contact area between the tubes and thus the van der Waals forces increases. Therefore the tensile strength and modulus were also increased. Liu et al [21] reported the tensile strength of aligned CNT/PVA composites based a praying-winding method was up to $1.8 \mathrm{GPa}$. Liu et al [22] extended the approach by adding a post hot-pressing process, and an epoxy solution was used to modify the CNT film. The hot-pressing method had been proved to be efficient in reducing the porosity of CNT film and benefit its tensile performance. The CNT/epoxy composite film exhibited a tensile strength and modulus of $1.54 \mathrm{GPa}$ and $59 \mathrm{GPa}$ respectively. It has been speculated that it is the capillary force that draws individual CNTs into closely packed CNT bundles, and works to improve CNT alignment degree, and the speculation also was verified in Liu's work. However, a certain degree of wavy CNTs in the aligned CNT composite film do not carry the load well and cannot be packed densely, and have poor inter-tube contacts, all of which adversely affect the strength, stiffness, and conductivity of resulting CNT composites. Wang et al [23] reported a novel stretch-winding process for fabricating high volume-fraction ( 46\%) CNT/polymer composites with a combined strength and Young's modulus that is not accessible to any current carbon fiber-reinforced polymer (CFRP) composites. When the CNT sheet was stretched by $12 \%$, a tensile strength of up to $3.8 \mathrm{GPa}$ and Young's modulus of up to $293 \mathrm{GPa}$ were achieved for the composite. The high loading, high orientation CNT composites have been fabricated through the newly fabricated technology, and the high tensile strength and modulus had been significantly improved than that of the obtained composites by CNT dispersion approach.

In the literature static mechanical properties of composites have been widely studied. 
However, the dynamic mechanical properties of composite material haven't been studied yet. Considering the composite materials are in a dynamic state in actual usage. We here used CNT array through the infiltration-winding approach to transfer vertically aligned CNT into horizontally aligned CNT film and also composited polyimide resin to prepare the aligned CNT/PI composites with high loading and a high degree of orientation for CNTs. Different CNT fraction of CNT/PI composites were obtained by tuning the concentration of PI solution, and their static and dynamic mechanical properties were also investigated. The results showed that the CNT/PI composites significantly improved the mechanical properties. The highest tensile strength and modulus of $3.9 \mathrm{GPa}$ and $182 \mathrm{GPa}$ were obtained by the $1 \mathrm{wt} \%$ concentration of PI solution, and the highest dynamic storage modulus was also obtained at the same condition. The glass transition temperature of PI increased over $130{ }^{\circ} \mathrm{C}$ due to the nanometer interface interaction between CNT and PI resin matrix. The CNT/PI composites demonstrated excellent structure stability. The excellent mechanical properties make the $\mathrm{CNT} / \mathrm{C}$ composites promising for many aerospace applications.

\section{Experimental}

\subsection{Composites preparation}

Vertically aligned CNT arrays used for the CNT film fabrication were synthesized at $750^{\circ} \mathrm{C}$ by chemical vapor deposition (CVD) on $\mathrm{SiO}_{2} / \mathrm{Si}$ substrate, which was coated with a $30 \mathrm{~nm}$ thick alumina and a $1 \mathrm{~nm}$ thick iron layer by an electro-beam evaporation technique. Argon with $6 \%$ hydrogen and pure ethylene were used as the forming gas and the carbon source, respectively. The total flow rate of gasses was set at $1.5 \mathrm{~L} / \mathrm{min}$. The grown CNTs were fewer-walled with a diameter of 4-5 nm, 2-3 walls, and $200 \mu \mathrm{m}$ in length. PI resin (ZKPI-320I, buy from POME Sci-tech Co., Ltd, Beijing) was used as the matrix system to composite with aligned CNT film. PI resin was dissolved in N-methylpyrrolidone (NMP), in which the weight fraction of PI was $0.1 \%, 0.5 \%, 1 \%, 2 \%, 4 \%$ and $6 \%$, respectively. Fig 1 shows schematically the infiltration-winding approach for fabricating CNT/PI precursor 
composites, where a horizontally continuous CNT sheet is drawn out of drawable vertically aligned CNT array and wound on a rotating polytetrafluoroethylene mandrel with constant winding speed. A pressure roller is installed closed to the rotating mandrel. And PI resin solution was dropped in the gap between the rotating mandrel and a pressure roller. On the one hand the pressure roller some pressure were applied for CNT film wrapped around the roller to increase the density of stacking film. On the other hand it can even dip resin solution on the film when the motor is driven roller rotation makes continuous CNT film pulled from the CNT array and layers wrapped around the mandrel. The thickness of all the CNT films was controlled by the number of winding revolutions. At last, the CNT/PI film was pressed under $4 \mathrm{MPa}$ for $140{ }^{\circ} \mathrm{C} 1 \mathrm{~h}, 190{ }^{\circ} \mathrm{C} 1 \mathrm{~h}, 220^{\circ} \mathrm{C} 2 \mathrm{~h}$, and $240{ }^{\circ} \mathrm{C} 3 \mathrm{~h}$ to cure and was further densified. Typical SEM images of CNT/PI films showing their surface morphologies are shown in Fig 2.

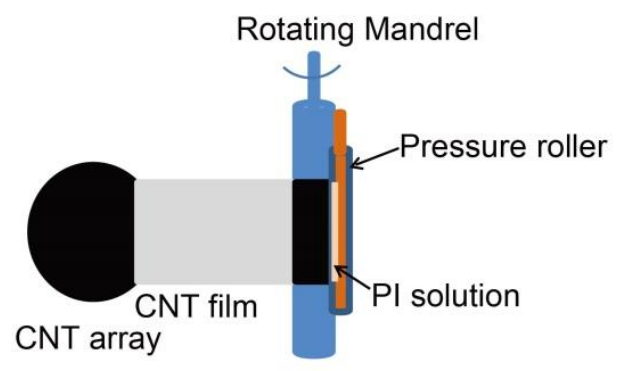

Fig 1 Schematic of the infiltration-winding preparation procedure for aligned CNT/PI composite film.
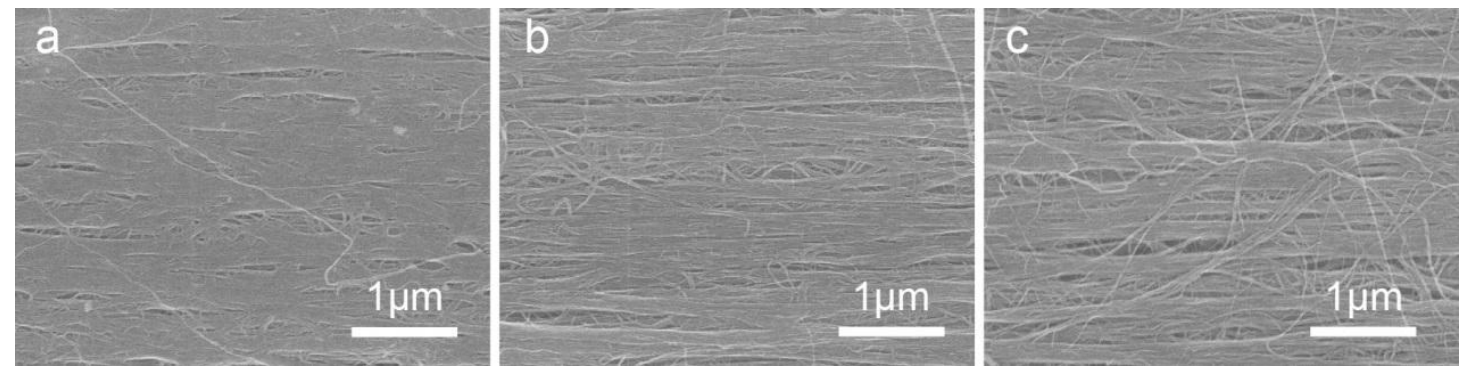

Fig 2 Typical SEM images of CNT/PI films showing their surface morphologies.

\subsection{Characterization and testing}

The morphology of CNT arrays, CNT films, and graphitized CNT films were 
characterized by scanning electron microscopy (SEM, Hitachi S-4800 HR-FESEM) and high-resolution transmission electron microscopy (HRTEM, FEI Tecnai G2 F20 S-Twin, $200 \mathrm{kV}$ ). The CNT weight fraction of CNT/PI composites was measured by thermo gravimetric analysis (TGA) conducted on a NETZSCH TG 209 F1 machine in nitrogen $(99.999 \%)$ with a heating rate of $10{ }^{\circ} \mathrm{C} / \mathrm{min}$ from $25{ }^{\circ} \mathrm{C}$ to $800{ }^{\circ} \mathrm{C}$. For tensile test, the CNT films were cut into strips with $25 \mathrm{~mm}$ in length and $\sim 1 \mathrm{~mm}$ in width parallel to the CNT alignment. The thickness and width of each strips were precisely measured by a micrometer and optical microscope, respectively. Mechanical testing was performed on a testing machine (Instron 3365) with a load cell of $100 \mathrm{~N}$ at a displacement rate of $0.5 \mathrm{~mm} / \mathrm{min}$. The gauge length was $6 \mathrm{~mm}$. At least five specimens were tested for each film.

The storage modulus, loss modulus, and tan of pure PI resin film and CNT/PI composites were measured at temperatures up to $550{ }^{\circ} \mathrm{C}$ in the tensile mode using a dynamic mechanical analyzer of the NETZSCH DMA 220. A heating rate of $3{ }^{\circ} \mathrm{C}$ /min was used. A frequency of $1 \mathrm{~Hz}$ was used and the oscillation amplitude used was $10 \mu \mathrm{m}$, with a specimen size of $15 \mathrm{~mm}$ in length and $1 \mathrm{~mm}$ in width and with the gauge length of $6 \mathrm{~mm}$.

\section{Results and discussion}

\subsection{Static mechanical properties}
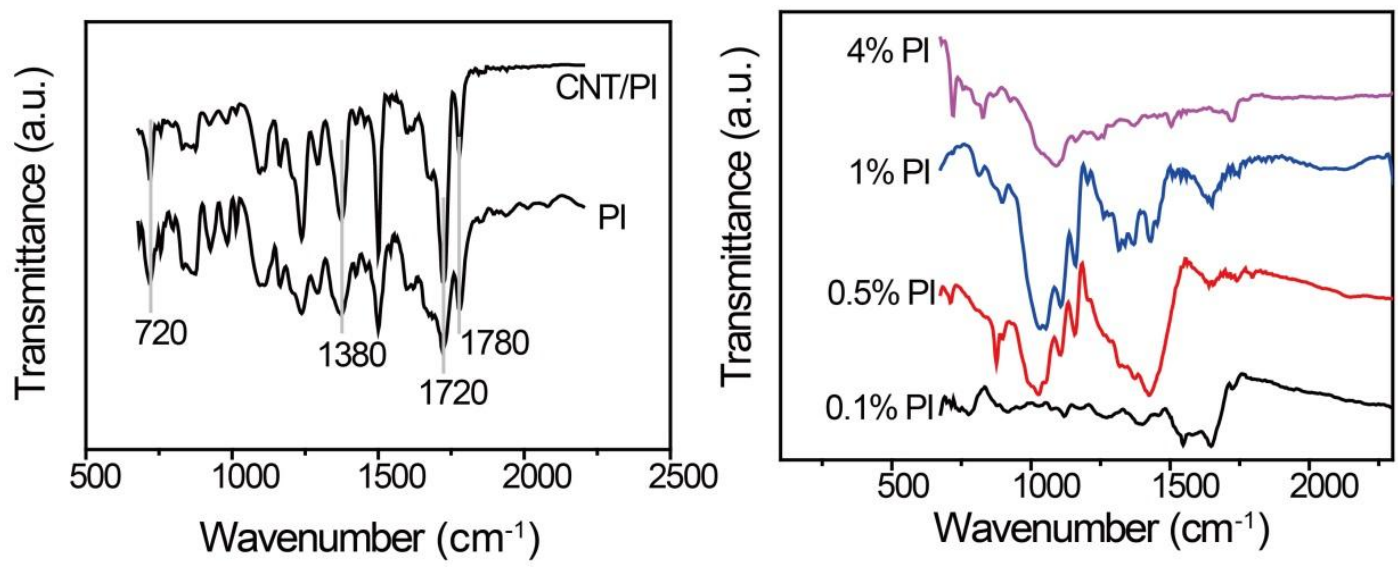

Fig 3 The FTIR of CNT/PI composites

The FTIR spectra of cured pure PI resin and CNT/PI composites were recorded to 
monitor the imidization. Different CNT/PI composites prepared by different concentration of PI resin solution after cured were characterized by FTIR. As shown in Fig 3, the imide absorption bands at $720 \mathrm{~cm}^{-1}$ (cyclic $\mathrm{C}=\mathrm{O}$ bending), $1380 \mathrm{~cm}^{-1}$, (C-N stretching), 1720 and $1780 \mathrm{~cm}^{-1}$ (symmetric and asymmetric $\mathrm{C}=\mathrm{O}$ stretching) is observed. CNT/PI compositesshowed characteristic peaks of PI, indicating that PI resin was successfully infiltrated into CNT films. The characteristic peaks of PI structure become more pronounced with the increase of PI concentration (Fig. 3b). .

Red shifts of the characteristic peaks of CNT/PI composites were observed comparaed to that of pure PI. and with the change of concentration the three peaks at $1720 \mathrm{~cm}^{-1}, 1380 \mathrm{~cm}^{-1}$, and $720 \mathrm{~cm}^{-1}$ have corresponding shifts. With the increasing concentration the peak of $1720 \mathrm{~cm}^{-1}$ blue shift, and the peak of $1720 \mathrm{~cm}^{-1}$ red shift, and the peak of $720 \mathrm{~cm}^{-1}$ blue shift. Because induced effects lead to peak appears blue shift, and conjugation effect can lead to a red shift. So it was deduced that the conjugation effect existed between the hexagon carbon ring of CNTs and benzene ring on the PI molecules. And induced effect also existed between CNTs and PI molecules during the PI curing process, which could lead to molecular structure of PI characteristic peak a red shift and blue shift phenomenon.

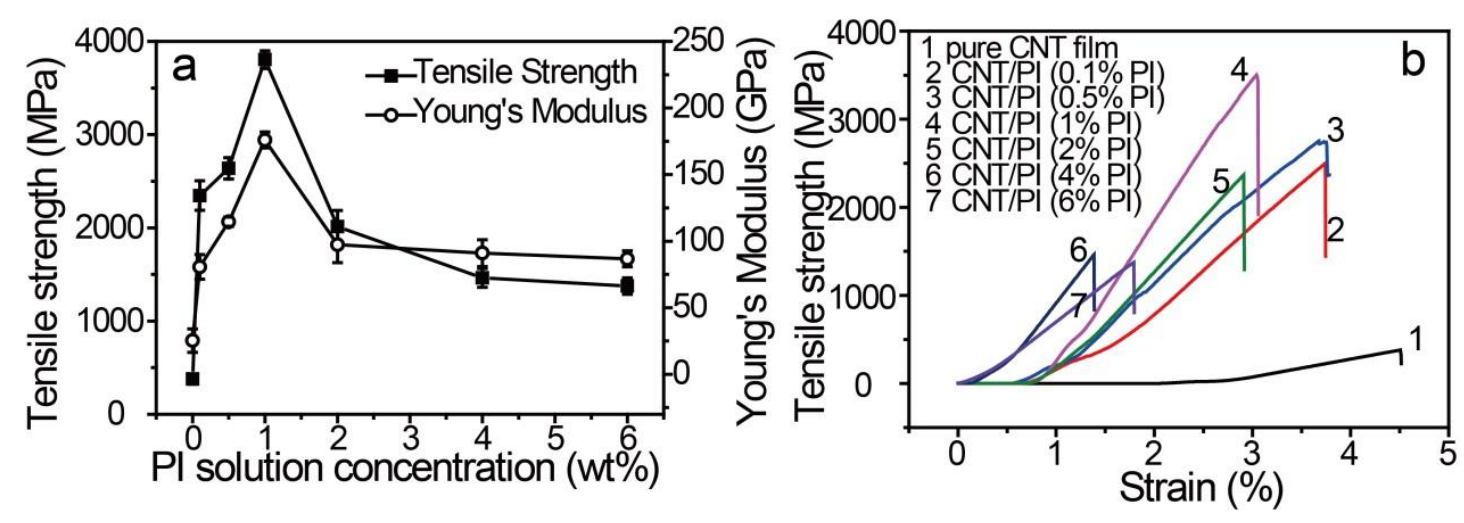




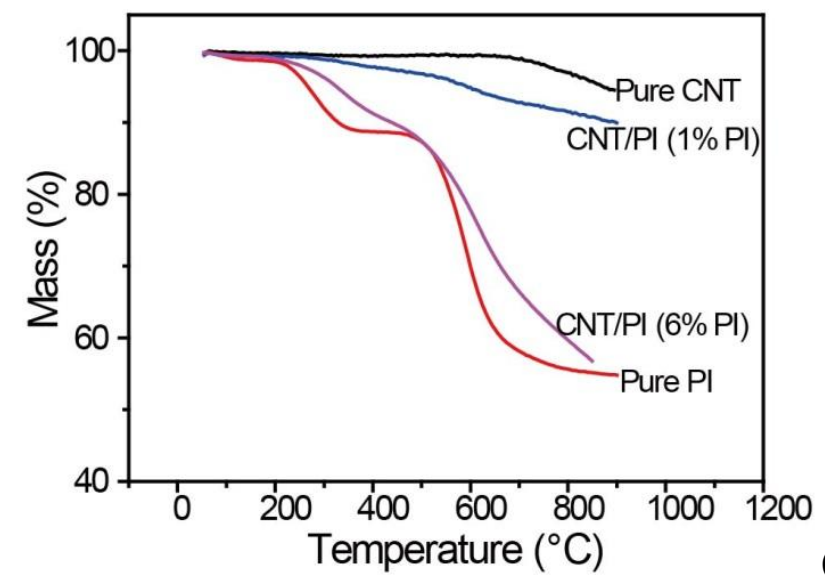

Fig 4 (a) The mechanical properties of CNT/PI composites as a function of PI solution concentration. (b) The stress-train curves of CNT/PI composites. (c) TGA curves for a pure CNT film, pure PI film and the CNT/PI composites prepared with the PI solution concentration of $1 \%$ and $6 \%$ respectively.

$\mathrm{CNT} / \mathrm{PI}$ composites having different CNT fraction were obtained changing the concentration of PI solutions, which includes $0.1,0.5,1,2,4$ and $6 \mathrm{wt} \%$. The static mechanical properties of pure CNT film and CNT/PI composites are shown in Fig 4. Pristine aligned CNT films without densification showed a tensile strength of $0.41 \mathrm{GPa}$ and a Young's modulus of 20.4GPa. The tensile mechanical properties were significantly improved after the CNT film composited with PI resin, and both were first increased and then decreased. The highest tensile strength and highest Young's modulus of $3.9 \mathrm{GPa}$ and $182 \mathrm{GPa}$ were obtained at the PI concentration of $1 \%$, which were increased by $951 \%$ and $839 \%$ compared with the pure CNT film, respectively. The tensile strength and modulus of the pure PI film are $138.61 \mathrm{MPa}$ and $2.2 \mathrm{GPa}$, respectively. Thus it can be seen that mechanical properties of composites are much higher than that of the each individual component, and the composite process played an obvious synergistic effect. As we know PI molecules containing a large number of benzene ring, which can adhere to CNT tubes through the $\pi$ - $\pi$ adsorption, therefore, the $\pi-\pi$ adsorption between CNTs lead to CNT film densified. The density of pure CNT film was $0.53 \mathrm{~g} / \mathrm{cm}^{3}$, and that of the composites corresponding to the $1 \% \mathrm{PI}$ solution was $1.23 \mathrm{~g} / \mathrm{cm}^{3}$. The film enhanced density thus lead to the enhanced CNT contact areas and subsequently the improved load transfer efficiency. At the same time, 
PI molecules cured and formed a cross-linked network which made the CNTs cling together, and made more tubes which carry load together at the same time. Thus the PI solution enhanced film strength, it did not only make CNT film shrunk to be densification, but also enhanced the bearing limitation.

The stress- strain curves of composites are shown in Fig 4(b). The pure CNT film without any densification and CNTs stacked layer by layer rely only on van der Waals interactions between tubes. There was not any polymer to limit its movement, so when applied load on it appeared larger slip between tubes, which in turn showed a large fracture strain. When CNT film composited with PI resin, PI resin cured and fixed the spatial location of CNTs, which limited the slippage between CNT tubes under the action of external load. Therefore, the CNT/PI composites showed lower fracture strain than the pure CNT film.

We also believed that the composites can get such a high performance was also attributed to the ideal CNT fraction in the composites. In general, the diluted polymer solution has low viscosity and is easy to be infiltrated into the CNT structures ${ }^{[24]}$, but PI and the CNT have their particular properties. Based on the TGA results shown in Fig 4(c), CNT mass fraction of composites prepared using 6\% PI solution is $10 \mathrm{wt} \%$, while the composites prepared using the $1 \%$ solution has $84.8 \mathrm{wt} \%$ CNTs, demonstrating that reduced the concentration of the PI solution can significantly improve the content of CNTs in composites. However, if further dilute the PI solution the CNT mass fraction will be increased, which results that PI matrix couldn't well infiltrate the CNTs. Thus the tensile strength of composites decreased. However, the tensile strength of composites using $6 \mathrm{wt} \%$ PI solution was also lower than that of $1 \%$ solution. That was because the CNT mass fraction was only $10 \mathrm{wt} \%$ at that time, and large amounts of rich resin, with a much lower strength of $0.128 \mathrm{GPa}$, was present between CNT tubes which leads to weakened the load transfers between CNT tubes. Therefore, the $1 \%$ concentration of PI solution was the optimized concentration for high strength composites.

The cross section morphologies of the tensile fracture surface of CNT/PI composites prepared by different concentration of PI solution are shown in Fig 5. It 
was found that at the low concentration of $0.1 \%$, CNTs assembled loose and there were large amounts of micro voids in the fracture surface. And it was also observed that the fracture surface showed multi-layer hierarchical structure due to insufficient PI resin to adhere CNTs tubes during the winding process. The CNT/PI composite film was prepared through a continuous CNT film was drawn from the spinning CNT array and wound onto the rorating mandrel. During the drawing process PI solution was sprayed onto the single layer film and stacked layer by layer. Thus when it was under the action of external force sliding was observed between layers. At the concentration of $1 \%$, the mass fraction of PI resin in the composites increased than that of $0.1 \%$ concentration. In the composites CNTs stacked closely and distributed uniformly, and no multi-layer structure. The uniformly distributed CNTs in the PI resin after PI resin cured formed a large network structure. At this condition PI resin filled between CNT tubes and adhered to CNT tubes, which lead to reduce the slide of CNTs. Therefore, the load transfer efficiency between CNTs increased. With higher PI concentration, the cross section of composites show multilayer fracture structure, and its layer thickness was greater than that of low concentration of $0.1 \%$. It can be observed that there was enough resin between CNTs from the high magnification SEM image shown in Fig 5(f). 

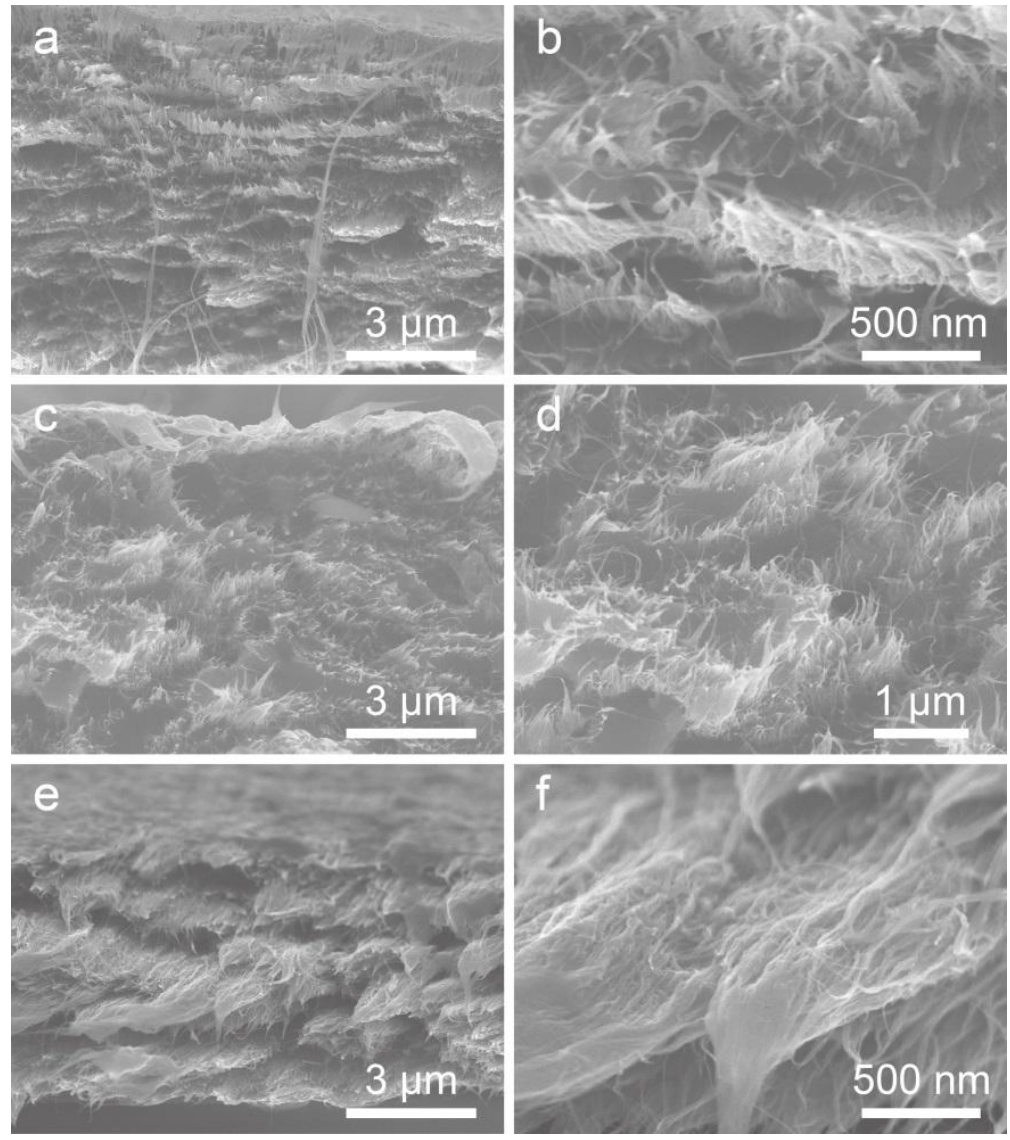

Fig 5 Low-(left column) and high-resolution(right column) SEM images of the fractures of composite films prepared using PI/NMP solutions having $0.1 \mathrm{wt} \%(\mathrm{a}, \mathrm{b})$, $1 \mathrm{wt} \%(\mathrm{c}, \mathrm{d})$, and $2 \mathrm{wt} \%(\mathrm{e}, \mathrm{f})$

\subsection{Dynamical mechanical properties}

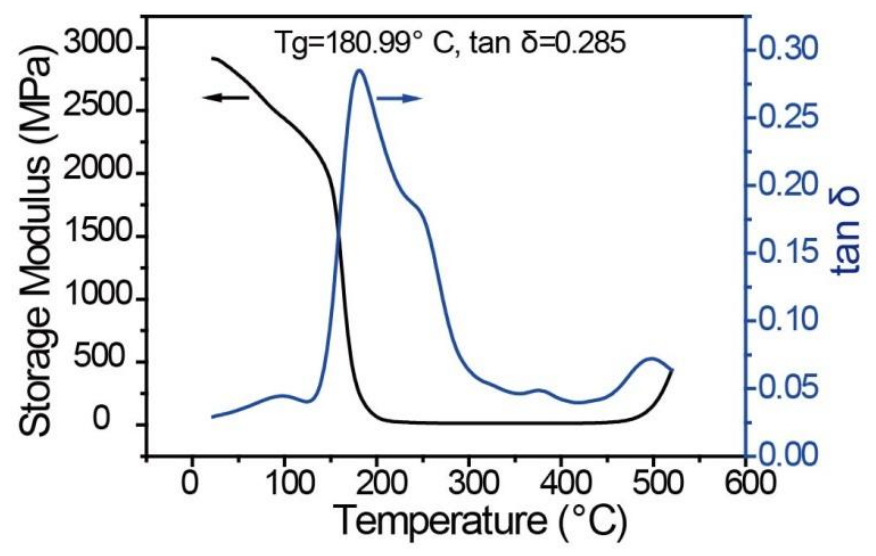

Fig 6 DMA test results of storage modulus and $\tan \delta$ of pure PI resin film.

Storage modulus refers to the material in the case of deformation, it stores energy due to the elastic deformation, which reflects the material's elasticity and characterizes the ability of resistance to dynamic deformation. Loss modulus is also 
called the viscous modulus, refers to the material in the case of deformation, it losses energy due to the viscous deformation, which reflects the material's viscosity. The results of dynamic properties of pure PI resin film are shown in Fig 6. Its storage modulus is $2.89 \mathrm{GPa}$ at the temperature of $30{ }^{\circ} \mathrm{C}$. The maximum of $\tan \delta$ is commonly taken as the glass transition temperature $\left(\mathrm{T}_{\mathrm{g}}\right)$. The $\mathrm{T}_{\mathrm{g}}$ of pure PI resin is $180.99^{\circ} \mathrm{C}$, and the maximum of $\tan \delta$ is 0.285 .

Fig 7 shows the dynamic properties of various CNT/PI composites as a function of temperature. Compared with Fig 6, it was found that Fig 7(a) the storage modulus of composites was two orders of magnitude higher than that the pure PI resin. The storage modulus of composites prepared with $0.1 \%$ PI solution was $160 \mathrm{GPa}$, and then with the increasing concentration of PI solution the storage modulus of composites at the $30{ }^{\circ} \mathrm{C}$ increased and then decreased. The highest storage modulus of composites of $224.55 \mathrm{GPa}$ at $30{ }^{\circ} \mathrm{C}$ was obtained at the concentration of $1 \%$. When the concentration was more than $1 \%$ the storage modulus of composites gradually decreased, illustrating that the elastic of materials reduced. While it was observed that the storage modulus of all the composites was higher than $135 \mathrm{GPa}$ and it was in agreement with the previous results of the static mechanical properties that the highest modulus was obtained at the PI concentration of $1 \%$. Combined with the TG results above, it shows that during the PI concentration from $6 \%$ to $0.1 \%$ the CNT mass fraction of composites gradually increased. And therefore the storage modulus of composites first increased and then decreased, analyzing the main reason may be: (1) CNT itself have excellent mechanical properties that made the composites show significantly improved storage modulus after CNT composite with PI resin. (2) As a result of the $\pi-\pi$ conjugation effect between PI molecules and CNTs, which make the PI had good compatibility with CNTs. Therefore the free volume of PI resin reduced and the PI macromolecular chain entangled with each other, which made the chain segment movement difficultly and greatly strengthened the interface bonding force. Thus the storage modulus of composites was significantly improved. (3) The reason may be that chemical bond formed between CNTs at their surface defects and PI molecules. The chemical bonds increased the storage modulus of the 
composites. As shown in Fig 7(b) and (c), the loss modulus and $\mathrm{T}_{\mathrm{g}}$ both were much higher than that of the pure PI matrix, it was supposed that secondary network structure was formed by the nanotubes in addition to the primary crosslink structure of polymers, and CNTs are immobilizing PI molecular chains at elevated temperature. CNTs as they made junction, internal friction of PI increases with the increasing temperature when PI molecular chains moved, thus the loss modulus increased. It was observed that the maximum value of $\tan \delta$ increased with the increasing concentration of PI solution, indicating that the stiffness of composites improved. And the maximum values of $\tan \delta$ all were compared with that of the pure PI resin film, because when CNT was introduced it reduced the effective volume of PI. Furthermore the internal friction mainly comes from the movement of the molecular chains, so the CNTs limited the movement of molecular chains and the tan $\delta$ peak values gradually reduced.
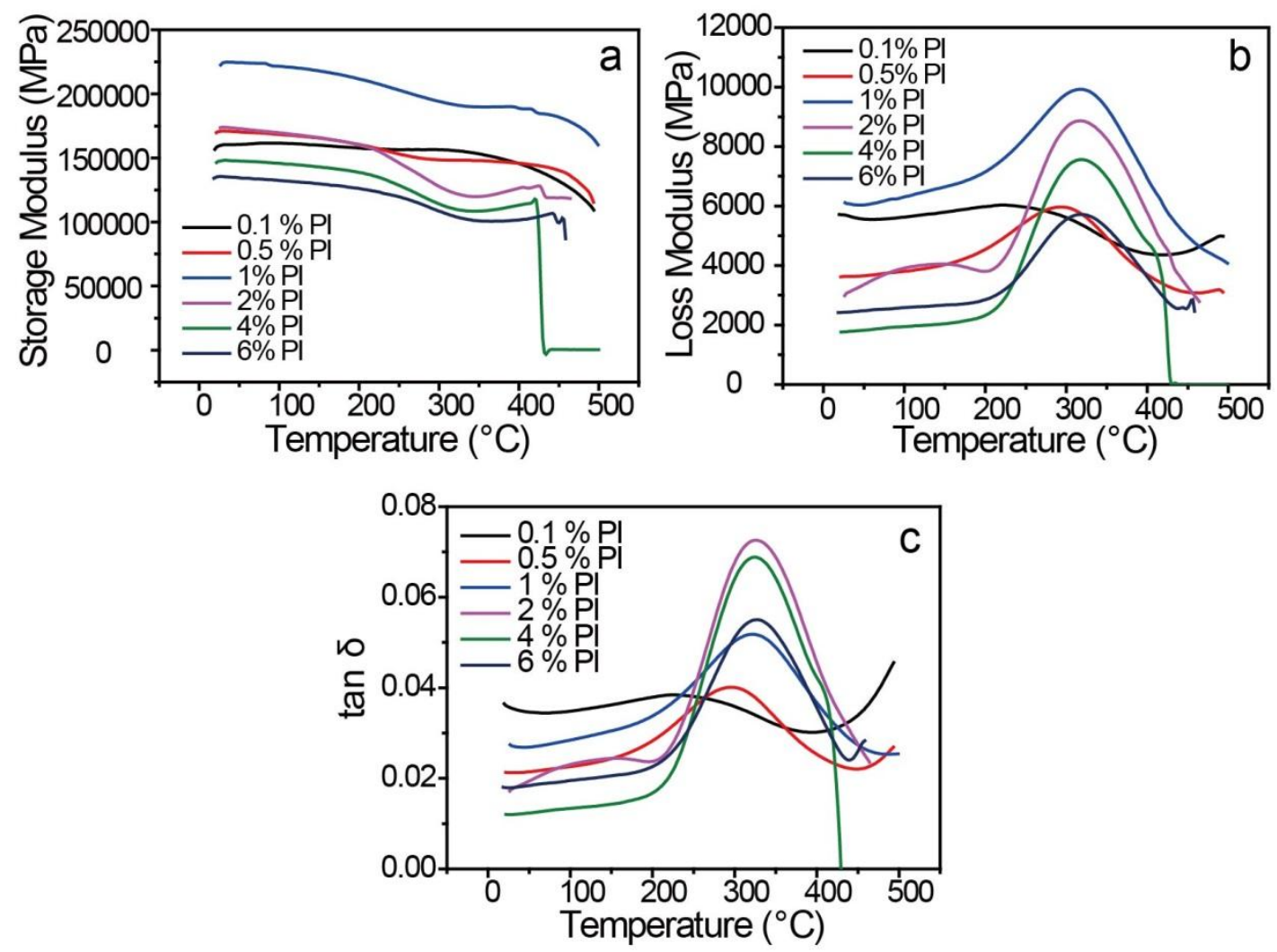

Fig 7 Dynamic properties of composites fabricated with different concentration of PI solution, (a) storage modulus, (b) loss modulus, and (c) $\tan \delta$ of the composites. 
The Tg results obtained from Fig 7(c) were summarized in Fig 8. The results show that with the increasing concentration of PI solution, the $T_{g}$ values increased and gradually shifted to the higher temperature, demonstrating that the heat resistance ability of CNT/PI composites increased. The Tg value of CNT/PI composites fabricated with $0.1 \%$ PI solution is $224.88{ }^{\circ} \mathrm{C}$, which is $43.89{ }^{\circ} \mathrm{C}$ higher than that of pure PI resin. And that of the CNT/PI composites fabricated using $0.5 \%$ PI solution is $295.8{ }^{\circ} \mathrm{C}$, which is $114.81{ }^{\circ} \mathrm{C}$ higher than that of the pure PI resin. And that of the CNT/PI composites fabricated using $1 \%$ PI solution is $321.69{ }^{\circ} \mathrm{C}$, further increase the concentration of PI solution the glass transition temperature of composites maintain at about a constant value of $325{ }^{\circ} \mathrm{C}$. Generally, the $\mathrm{Tg}$ of composites modified by filling CNTs whose content usually less than $10 \mathrm{wt} \%$ was increased by only a few degree Celsius, that of the composites modified by functionalized CNTs such as the carboxylic CNT composites appeared dozens of degrees Celsius rise. In our work, there was significantly enhancement of $146^{\circ} \mathrm{C}$ for Tg value of the CNT/PI composites which was the aligned CNT film coupling with PI resin. So this increase demonstrated that there were strong interactions between CNTs and PI molecules, which made the interface combination between CNTs and PI stronger. Therefore the composites with improved interface strength under external force can effectively transfer the load to the CNTs. Thus there was a significant improvement in the mechanical properties.

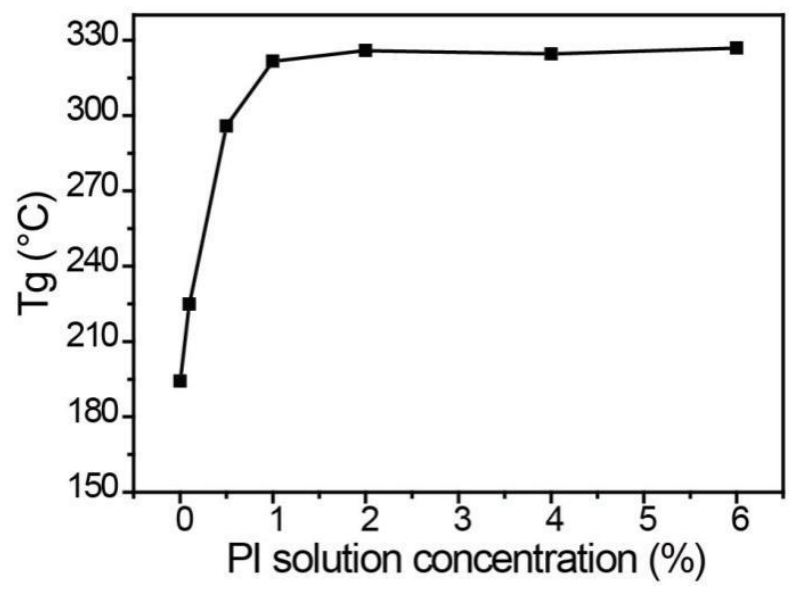

Fig 8 The glass transition temperature $\left(\mathrm{T}_{\mathrm{g}}\right)$ of the $\mathrm{CNT} / \mathrm{PI}$ composites as a function of 
the concentration of PI solution.

In our present work, the CNT/PI composites were fabricated through layer by layer assembly. The static and dynamic mechanical properties showed that they had excellent mechanical performance. Further, with the highest mechanical properties of CNT/PI composites fabricated using $1 \%$ concentration of PI solution were conducted by DMA test at $25{ }^{\circ} \mathrm{C}$ with a frequency of $1 \mathrm{~Hz}$ to characterize its structural stability. The results were shown in Fig 9. Within continuous 96 hours testing process, the storage modulus of composites was maintained a constant value over $200 \mathrm{GPa}$, which illustrated that the internal structure of composites kept stability without damage. The storage modulus of the initial stage was amplified shown in Fig 9(b), which can be seen that the storage modulus in the first 300 seconds increased slightly and then remains constant. It was also called the strain hardening phenomenon due to the wavy CNT tubes were stretched and orientated. Therefore, the CNT/PI composites showed well structure stability.
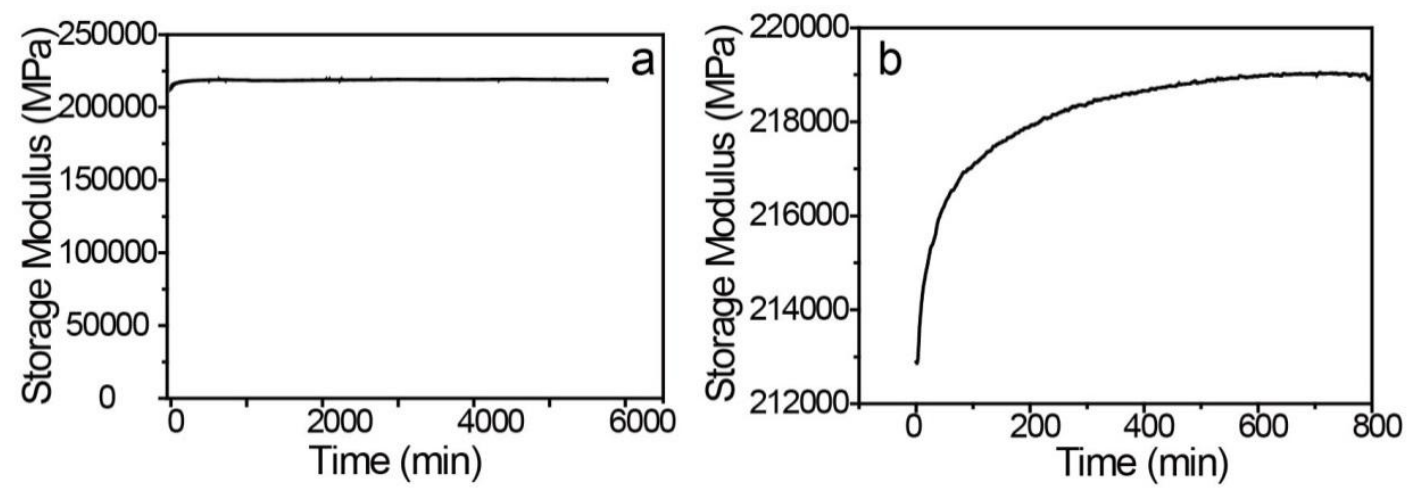

Fig 9 The storage modulus of CNT/PI composite versus time under constant temperature.

\section{Conclusions}

We have fabricated ultrastrong and excellent dynamic mechanical properties of CNT/PI composites with a high degree of orientation of CNTs by the infiltration-winding method to make the aligned few walled CNT film composite with PI resin by using a layer by layer assembly. PI solution had significant densification effect on the CNT film, which can enhance the contact area of CNT tubes and 
improved the interaction between CNTs. And these composites with a high mass fraction of long and well aligned CNTs that uniform distributed in the PI resin. The highest tensile strength and modulus of CNT/PI composites were obtained at the PI concentration of $1 \%$, which were $3.9 \mathrm{GPa}$ and $182 \mathrm{GPa}$, respectively. And also these composites showed high dynamic performance and good structural stability. The DMA results showed that these composites have high temperature resistance up to $325{ }^{\circ} \mathrm{C}$. These composites have the potential to be used as the structural and functional materials at high temperature in the aerospace field.

\section{Acknowledgement}

The authors acknowledge the financial supports from the Chang Jiang Scholars Program and National Science Foundation of China (Grant Number 21273269, 21473238, 51675095 and 11572085). The financial supports from Foundation for the

Fok Ying-Tong Education Foundation (Grant No. 141070), Shu-Guang project (Grant No. 14SG31) supported by Shanghai Municipal Education Commission and Shanghai Education Development Foundation, the Fundamental Research Funds for the Central Universities of China and DHU Distinguished Young Professor Program are also gratefully acknowledged.

\section{References}

[1] Robert IM. Advanced composite structures research in Australia. Composite Structures, 2002, 57: 3-10.

[1] Njuguna J, Pielichowski K, and Desai S. Nanofiller-reinforced polymer nanocomposites. Polym. Adv. Technol., 2008, 19: 947-959.

[2] Cury C, Pedro H, Satyanarayana, KG, et al. Nanocomposites: Synthesis, Structure, Properties and New Application Opportunities. Mat. Res., 2009, 12(1): 1-39.

[3] Leng J, Lu, H; Liu, Y, et al. Shape-Memory Polymers-A Class of Novel Smart Materials. MRS Bulletin, 2009, 34(11): 848-855. 
[4] Iijima S. Helical microtubules of graphitic carbon. Nature, 1991, 354: 56-58.

[5] Kim JA, Seong DG, Kang TJ, et al., Effects of surface modification on rheological and mechanical properties of CNT/epoxy composites. Carbon, 2006, 44(10): $1898-1905$.

[6] Spitalsky Z, Tasis D, Papagelis K, et al., Carbon nanotube-polymer composites: chemistry, processing, mechanical and electrical properties. Progress in Polymer Science, 2010, 35(3): 357-401.

[7] Dresselhaus MS, Dresselhaus G, et al., Science of Fullerenes and Carbon Nanotubes. Academic: San Diego, CA 1996.

[8] Treacy MMJ, Ebbesen TW, and Gibson JM. Exceptionally high Young's modulus observed for individual carbon nanotubes. Nature, 1996, 381(6584): 678-680.

[9] Ebbesen TW, Lezec HJ, Hiura H, et al. Electrical conductivity of individual carbon nanotubes. Nature, 1996, 382(6586): 54-56.

[10]Berber S, Kwon YK, and Tomanek D. Unusually high thermal conductivity of carbon nanotubes. Phys. Rev. Lett., 2000, 84(20): 4613-4616.

[11]Zeng ZH, Jin $\mathrm{H}$, Zhang LP, et al. Low-voltage and high-performance electrothermal actuator based on multi-walled carbon nanotube/polymer composites. Carbon, 84, April 2015, 327-334.

[12] Ajayi OA, Guitierrez DH, Peaslee D, et al. Electrophoretically deposited graphene oxide and carbon nanotube composite for electrochemical capacitors. Nanotechnology, 2015, 26(41): 415203.

[13] Islam MS, Deng Y, Tong LY, et al. Grafting carbon nanotubes directly onto carbon fibers for superior mechanical stability: Towards next generation aerospace composites and energy storage applications. Carbon, 2016, 96: 701-710.

[14] Hong SK, Kim D, Lee S, et al. Enhanced thermal and mechanical properties of carbon nanotube composites through the use of functionalized CNT-reactive polymer linkages and three-roll milling. Composites Part A: Applied Science and Manufacturing, 2015, 77: 142-146.

[15] Micheli D, Vricella A, Pastore A, et al. Ballistic and electromagnetic shielding behaviour of multifunctional Kevlar fiber reinforced epoxy composites modified by 
carbon nanotubes. Carbon, 2016, 104: 141-156.

[16] Kaseem M, Hamad K, and Ko YG. Fabrication and materials properties of polystyrene/carbon nanotube (PS/CNT) composites: A review. European Polymer Journal, 2016, 79: 36-62.

[17] Hong JS, Lee JH, and Nam YW. Dispersion of solvent-wet carbon nanotubes for electrical CNT/polydimethylsiloxane composite. Carbon, 2013, 61: 577-584.

[18] Liu P, Lam A, Fan Z, Tran TQ, Duong HM. Advanced multifunctional properties of aligned carbon nanotube-epoxy thin film composites. Materials and Design,2015,87:600-605.

[19] Tran TQ, Fan Z, Liu P, Lam A, Duong HM. Advanced morphology-controlled manufacturing of carbon nanotube fibers,thin films and aerogels from aerogel technique. Asia Pacific Confederation of Chemical Engineering Congress 2015.

[20] Duong HM, Gong F, Liu P, Tran TQ. Advanced Fabrication and peoperties of aligned carbon nanotube composites: Experiements and Modeling. Carbon Nanotube - Current Progress of their Polymer Composites 2016-Intech.

[21] Liu W, Zhang $\mathrm{XH}, \mathrm{Xu} \mathrm{G}$, et al. Producing superior composites by winding carbon nanotubes onto a mandrel under a poly(vinyl alcohol) spray. Carbon. 2011, 49(14):4786-91.

[22] Liu YN, Li M, Gu YZ, et al. Ultrastrong carbon nanotube/ bismaleimide composite film with super-aligned and tightly packing structure. Composites Science and Technology, 2015, 117(29): 176-182.

[23] Wang X, Yong ZZ, Li QW, et al. Ultrastrong, stiff and multifunctional carbon nanotube composites. Materials Research Letters. 2013, 1(1): 19-25

[24] Thang Q, Zeng F, Anastaslia M, et al. Post-treatments for Multifunctional Property Enhancement of Carbon Nanotube Fibers from the Floating Catalyst Method. ACS Applied Materials \& Interfaces., 2016, 8(12): 7948-7956. 